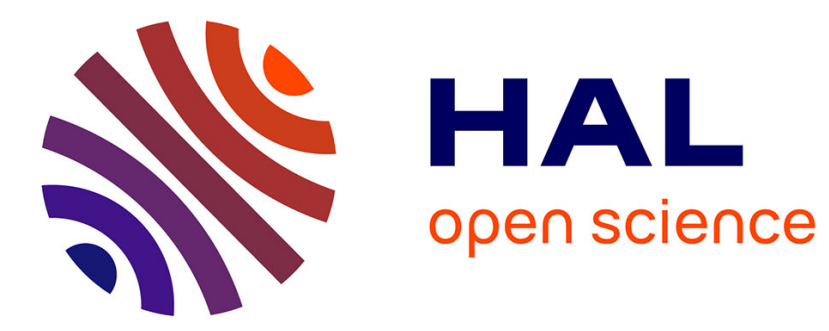

\title{
La prescription conditionnelle, un espace de liberté en trompe l'œil!
}

\author{
A. Rolland, N. Goutte, P. Viala
}

\section{To cite this version:}

A. Rolland, N. Goutte, P. Viala. La prescription conditionnelle, un espace de liberté en trompe l'œil !. NPG: Neurologie - Psychiatrie - Gériatrie, 2019, 19, pp.99 - 104. 10.1016/j.npg.2018.12.005 . hal-03486318

\section{HAL Id: hal-03486318 \\ https://hal.science/hal-03486318}

Submitted on 21 Dec 2021

HAL is a multi-disciplinary open access archive for the deposit and dissemination of scientific research documents, whether they are published or not. The documents may come from teaching and research institutions in France or abroad, or from public or private research centers.
L'archive ouverte pluridisciplinaire HAL, est destinée au dépôt et à la diffusion de documents scientifiques de niveau recherche, publiés ou non, émanant des établissements d'enseignement et de recherche français ou étrangers, des laboratoires publics ou privés.

\section{다)(1) $(5$}

Distributed under a Creative Commons Attribution - NonCommerciall 4.0 International 


\title{
La prescription conditionnelle, un espace de liberté en trompe l'œil !
}

\author{
"As needed" prescription: an optical illusion of freedom to act!
}

\author{
A. Rolland ${ }^{1}$, N. Goutté ${ }^{2}$, P. Viala ${ }^{3}$
}

1-Infirmière, SSR Polypathologie, pôle de gériatrie, Hôpital Universitaire Paul Brousse (HUPS, AP-HP), 14 avenue Paul Vaillant Couturier, 94800 Villejuif. agnes83rolland@gmail.com

2-, PhD RN, Coordonateur de la recherche paramédicale Hôpitaux Universitaires Sud (HUPS), DHU Hépatinov, INSERM UMRS 1193, Hôpital Paul Brousse, Direction des soins, 14 avenue Paul Vaillant Couturier, 94800 Villejuif. nathalie.goutte@aphp.fr

3-Cadre de santé, SSR Polypathologie, pôle de gériatrie, Hôpital Universitaire Paul Brousse (HUPS, AP-HP), 14 avenue Paul Vaillant Couturier, 94800 Villejuif. Pascale.viala@aphp.fr

Auteure correspondante : A. Rolland agnes83rolland@gmail.com 


\section{Résumé}

Ce texte aborde la prescription conditionnelle ou en «si besoin » et fait une présentation des textes législatifs et des recommandations qui encadrent les pratiques médicales, pharmaceutiques et soignantes notamment celles des infirmiers. Au-delà de cet aspect règlementaire, il est nécessaire de renforcer le travail d'équipe tout en maintenant une autonomie des soignants dans le but d'améliorer et d'harmoniser les pratiques paramédicales et médicales.

Mots-clés : prescription; recommandation de bonne pratique; texte législatif ; pratiques soignantes

\section{Summary}

This paper addresses the question of conditional or "as needed" prescription and presents the legal texts and recommendations relating to medical, pharmaceutical and caregiver practices, particularly those of nurses. Beyond this regulatory aspect team work needs to be reinforced while at the same time maintaining the autonomy of caregivers in order to improve and harmonize paramedical and medical practices.

Keywords: prescription; good practice recommendation; legislative text; caregiver practice 
La prise en charge médicale à l'hôpital évolue, notamment en ce qui concerne les prescriptions médicamenteuses. En particulier, la diminution du nombre de médicaments prescrits est encouragée par les pouvoirs publics et sanitaires. En même temps, les mesures visant à assurer une plus grande sécurité de la prise en charge médicamenteuse des patients sont de plus en plus importantes. L'objectif est une utilisation sécurisée, appropriée et efficiente des médicaments à l'hôpital [1]. Une partie de ces objectifs pourrait être atteinte à l'aide des prescriptions informatisées. Dans une étude concernant le circuit du médicament en établissement hospitalier, Meyer et al [2] ont montré que 6,8\% des médicaments prescrits ne sont pas administrés alors que $10,1 \%$ de médicaments non prescrits sont donnés. Cela concerne principalement des médicaments à faible activité thérapeutique.

Parmi les prescriptions potentiellement à risque, on peut identifier la prescription conditionnelle ou en «si besoin ». Selon la Direction de l'hospitalisation et de l'organisation des soins [3] : "Est appelée prescription conditionnelle la prescription d'un médicament en dose variable en fonction de l'évaluation d'un ou plusieurs paramètres cliniques et/ou biologiques pour un patient donné. Le champ de ce type de prescription est clairement défini en concertation par les équipes médicales et infirmières et validé par la COMEDIMS (Commission du médicament et des dispositifs médicaux stériles). Les paramètres d'adaptation sont très clairement identifiés. La prescription comporte le nom du médicament, sa voie d'administration, la posologie ainsi que la durée de validité qui est limitée. »

Une enquête sur les pratiques professionnelles auprès des équipes officinales de Lorraine [4] a montré que les antalgiques représentent $47 \%$ des prescriptions conditionnelles, $14 \%$ pour les laxatifs et un nombre important pour les anxiolytiques. Dans les services de soins de suite et de réadaptation (SSR) du pôle de gériatrie de l'hôpital Paul Brousse, 68\% des prescriptions comportent au moins une prescription conditionnelle.

L'utilisation de la prescription conditionnelle chez la personne âgée pose plusieurs questions : quel est le rôle de l'infirmière en termes de pratiques professionnelles ? Dans cette espace d'autonomie, comment est assurée la sécurité du patient ? Comment prendre de la distance par rapport à une situation afin de conserver un regard objectif ? Qu'est-ce qui est nécessaire à l'équipe soignante pour analyser une situation?

Afin de mener une analyse sur la prescription conditionnelle et le rôle de l'infirmière, nous nous sommes interrogés sur le cadre législatif, officiel de la prescription conditionnelle, tant au niveau de la prescription du médicament que dans son administration. Pour nous aider dans notre réflexion, nous prendrons en compte les recommandations de bonnes pratiques. Nous reprendrons aussi les décrets du travail de l'infirmier. Cela nous conduira à rappeler aux professionnels de santé les pratiques soignantes à adopter et les risques à éviter au regard de la prescription conditionnelle.

\section{Recommandations concernant la prescription conditionnelle}

La sécurité et la qualité de la prise en charge médicamenteuse impliquent différents acteurs : médecins, pharmaciens et infirmiers.

\section{$\underline{\text { Les médecins }}$}

La Direction générale de l'offre de soins (DGOS) dans son texte : "Qualité de la prise en charge médicamenteuse ; Outils pour les établissements de santé »[5] indique les étapes à 
réaliser en vue d'une prescription adaptée à chaque patient. Dans ce texte, il est expliqué que la prescription conditionnelle vient après plusieurs étapes qui sont : en premier l'évaluation du traitement initial, puis l'évaluation de l'autonomie et de l'observance des traitements, et l'apport de l'entourage. La prescription conditionnelle fait suite à l'évaluation régulière du traitement.

Comme le stipule le Code de Santé publique dans son article R4127-8, la prescription est adaptée aux circonstances [6]. Au-delà de la liberté de prescrire, les médecins selon l'article R4127-8 du Code de Santé publique ont deux exigences à respecter : lisibilité et clarté en vue de la bonne exécution de la prescription. Elle permet aussi de construire un langage commun entre les médecins et les soignants.

\section{Les pharmaciens}

L'Article R4235-48 du Code de Santé publique annonce les obligations du pharmacien [7] :

- la mise à disposition des informations et les conseils nécessaires au bon usage du médicament ;

- il a un devoir particulier de conseil lorsqu'il est amené à délivrer un médicament qui ne requiert pas une prescription médicale ;

- il doit, par des conseils appropriés et dans le domaine de ses compétences, participer au soutien apporté au patient.

Il est stipulé dans ce même article [7] que les médicaments peuvent être délivrés globalement à l'unité de soins en renouvellement d'une dotation adaptée, préalablement définie par le pharmacien et le médecin responsable de l'unité de soins. L'outil informatique permet à la pharmacie à usage intérieur l'accès aux informations nécessaires à l'analyse pharmaceutique des prescriptions.

\section{$\underline{\text { Les infirmiers }}$}

Il s'agit ici d'aborder la règlementation de la pratique de la profession d'infirmier, et plus précisément, les Actes professionnels-exercices de la profession [8].

Dans les articles suivants sont indiqués ce que les infirmiers doivent exécuter :

Art.R4311-2 «De contribuer à la mise en cuvre des traitements en participant à la surveillance clinique et à l'application des prescriptions médicales contenues, le cas échéant dans des protocoles établis à l'initiative du ou des médecins prescripteurs. »

Art.R4311-5 «Aide à la prise des médicaments non injectables. Vérification de leur prise. Surveillance de leurs effets et éducation du patient. »

Art.R4311-8 «L'infirmier ou infirmière est habilité à entreprendre et à adapter les traitements antalgiques, dans le cadre des protocoles préétablis, écrits, datés et signés par un médecin. Le protocole est intégré dans le dossier de soins infirmiers. »

La dispensation des médicaments est régie par l'arrêté du 6 avril 2011 [1] et la circulaire du 14 février 2012 [9] relatifs au management de la qualité de la prise en charge médicamenteuse et au médicament dans les établissements de santé. 
Le travail en collaboration nécessite de transmettre les informations oralement et par écrit aux professionnels de santé et aux patients selon le Référentiel d'activités de l'arrêté du 31 juillet 2009 [10].

Afin d'éviter les interprétations des prescriptions et d'assurer une qualité optimale dans la prise en charge médicamenteuse les infirmiers sont invités à réaliser des recherches et veilles professionnelles. La réalisation de bilan des pratiques professionnelles avec les pairs d'une même profession ou avec d'autres professionnels de la santé est encouragée [10].

\section{Discussion}

D'un point de vue juridique

Comme le souligne de Benalcazar [11] "l'infirmier n'est pas autorisé à interpréter les symptômes », son rôle est d'administrer les traitements et non de décider de l'opportunité de celui-ci. Il rappelle que le "risque peut provenir d'un mauvais diagnostic médical ou de l'inopportunité de l'administration d'un traitement ».

Ce même auteur, docteur en droit, évoque le fait que les pouvoirs publics ont pris en compte la prescription en "si besoin». Il stipule que "les établissements de santé sont aux prises avec des injonctions paradoxales avec des exigences légales et règlementaires rigoureuses et pointilleuses lorsqu'il s'agit de la prise en charge du patient - de la prescription jusqu'à l'administration - et des contraintes organisationnelles et opérationnelles fortes qui exigent une certaine souplesse. La conséquence de ces injections paradoxales est la pratique du "si besoin ", dont la qualité juridique se révèle difficile à opérer sans que pour autant le risque soit très élevé. » [11]

\section{$\underline{\text { Autonomie des soignants }}$}

Si le personnel infirmier a acquis de l'autonomie à travers son rôle propre, la prescription conditionnelle lui offre une occasion d'explorer et d'appliquer l'étendue de ses pratiques et connaissances. C'est dans cet aspect que l'on peut mesurer les limites de l'exercice infirmier, mais aussi les terrains à explorer nouveaux ou futurs.

L'autonomie a plusieurs sens et peu se définir comme la capacité à faire des choix. Selon Zarifian [12], «l'autonomie est une condition incontournable d'un déploiement de la compétence ». La compétence n'existe que si l'acteur a ou se donne une marge d'initiative et de décision et ne se borne pas à suivre des prescriptions [12]. La notion d'autonomie renvoie alors et englobe les notions de capacité, d'aptitude et de processus [13].

Dès lors, différents aspects viennent conditionner le choix de la prescription en «si besoin ». Tout d'abord, il y a la question de la faisabilité et de la capacité à intervenir $24 \mathrm{~h} / 24$. Cette prescription doit pouvoir couvrir les besoins du patient à tout moment. D'autre part, la polypathologie des personnes âgées, responsable d'une polymédication importante, implique d'essayer de limiter le plus possible le nombre de molécules et de prises. La prescription conditionnelle peut y aider. Elle est d'ailleurs souvent utilisée de façon transitoire avant l'arrêt définitif d'un traitement symptomatique. Cette prescription contribue à la pratique réflexive par rapport aux soins. Cette démarche réflexive est encouragée par les équipes médicales qui laissent alors une liberté de gestion au personnel infirmier [4]. 


\section{Un cadre à reconstruire}

Si certaines prescriptions ne définissent pas le choix de l'administration du médicament, elles invitent les soignants à redéfinir un cadre de pratique de soins. Par exemple, une prescription prévoit : administrer 1 comprimé 5mg d'Oxynormoro toutes les 6 heures si douleur. D'une part, le soignant ne peut se contenter de donner un antalgique à la demande du patient. Les soignants doivent choisir une échelle d'évaluation adaptée qui soit commune à tous pour éviter toute subjectivité. Ils connaissent les scores à partir desquels on considère la douleur d'un patient. Ils recherchent lors de l'évaluation quand cela est possible : le type de douleur, son intensité, le début, sa durée, les positions antalgiques s'il y en a.

D'autre part, on souhaiterait lire plus souvent en complément d'une prescription conditionnelle en lien avec la douleur : administrer si douleur égale ou supérieure à $2 / 5$ pour l'échelle Algoplus, par exemple, qui est fréquemment utilisée dans les services de gériatrie.

Toutes ces informations offrent un cadre d'intervention pour les soignants qui sécurise les soins. Cette autonomie laissée aux soignants incite à plus de coopération et de communication afin d'assurer une prise en charge de qualité. En effet pour certains auteurs [14], la communication et les stratégies de réflexion collectives sont déterminantes au bon déroulement des soins.

Chator [4] dans son travail de recherche présente des propositions de bonnes pratiques et de recommandations professionnelles qui sont résumées sur le tableau 1.

Ces recommandations sont d'autant plus pertinentes que dans l'administration des traitements pour la personne âgée il faut prendre en compte l'état de la fonction rénale, hépatique, et le fait que la prescription à une personne compte plusieurs médicaments ou non. Il est également recommandé par l'Agence Nationale de Sécurité du Médicament (ANSM) [15] de diminuer les dosages et d'espacer les prises pour certains traitements.

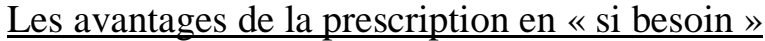

La prescription conditionnelle permet d'anticiper sur les situations susceptibles d'être rencontrées. Elle rassure les soignants qui peuvent alors plus facilement faire face à des situations complexes. Elle leur laisse une autonomie de raisonnement et une autonomie pour l'évaluation des situations plus importantes.

Il est ainsi parfois possible en se référant aux données probantes [16], par exemple, d'anticiper sur les événements en prévoyant des prescriptions conditionnelles. Pour les soignants, ce type de prescription permet d'intervenir rapidement au regard des symptômes à un moment donné. En effet, le fait de s'appuyer sur des preuves renforce la pratique clinique [17].

Ce type de prescription offre plus de souplesse dans les interventions des soignants vis-à-vis des patients âgés et peut aider à une meilleure adhésion aux traitements. Par exemple lorsqu'un malade douloureux prend conscience de l'efficacité de son traitement antalgique. Bien souvent le patient devient alors partenaire du soin en demandant les traitements dont il ressent réellement le besoin. 
Cette prescription en «si besoin » autorise également le refus de la personne âgée (et donc son autonomie), ou de remettre à plus tard une décision. Cela permet de remettre le malade au centre de la réflexion des soignants.

\section{Des exigences à respecter}

La continuité de la prise en charge passe aussi par l'analyse des prescriptions et des situations cliniques au cours du parcours de soins [18]. La traçabilité et les différentes transmissions prennent alors tous leurs sens [19]. Une grande partie de la réussite d'une prise en charge repose sur la communication orale entre les différents professionnels qui interviennent auprès des patients afin de réajuster sans cesse les prises en charge [14]. C'est pourquoi pour nous aider à harmoniser nos pratiques, nous avons des moments de communications orales notamment pendant les transmissions inter équipes [20]. Ces instants-là sont souvent l'occasion pour les soignants d'évoquer les difficultés, les évolutions au regard d'un état de santé. Ces transmissions permettent "de donner le change, de réagir, de déclencher la confrontation des versions de la réalité » [21], et d'évacuer les erreurs personnelles [22].

Une fois par semaine, nous avons également une réunion pluriprofessionnelle avec l'ensemble des professionnels de santé (médecins, infirmiers, aides-soignants, cadre, etc.) pour dresser le bilan des prises en charges individuelles.

La justification des soignants dans l'administration des thérapeutiques en «si besoin »vient expliquer leurs choix qui peuvent s'avérer judicieux en fonction d'une situation, d'un moment de la journée, et de la personne. La collaboration entre professionnels implique de l'autonomie, un effort intellectuel, un but, et une signification partagée par ces derniers [20].

Si la prescription conditionnelle permet une souplesse d'intervention, elle nécessite, et on insiste, un investissement important. Il semble que la prescription conditionnelle soit bénéfique dans le sens où le soignant est bien souvent la personne qui côtoie le plus les patients et qui analyse les situations cliniques avec une prise de décision complexe et dynamique [23]. Pour renforcer l'idée d'investissement, certains auteurs expliquent le lien étroit entre l'engagement, l'autonomie mais aussi le soutien social que l'on entend comme construction collective. En effet, il apparaît que le soutien social renforce la relation entre autonomie et travail d'engagement [24]. L'effet se mesure directement sur le patient et dans le travail de l'équipe. Des auteurs comme Wade [25] démontrent l'engagement responsable des soignants dans les équipes de soins. Ils expliquent également qu'une amélioration au niveau des équipes [26] est constatée lorsqu'il y a un partage des pratiques entre les membres producteurs de soins.

\section{$\underline{\text { Pour une pluridisciplinarité }}$}

Comme nous l'avons signalé en introduction, $10,1 \%$ de médicaments non prescrits sont administrés [2]. Face à ce constat, on ne peut faire l'économie de rappeler l'importance des recommandations de la Haute Autorité de la Santé (HAS), des sociétés savantes, et autres autorités en ce qui concerne la prise en charge médicamenteuse selon le profil des patients, mais également en fonction des traitements.

Le texte de la HAS [27] concernant la méthodologie de la prescription stipule au regard de la qualité de la prescription médicale qu' «on distingue trois sortes de problèmes associés à la prescription de médicaments chez la personne âgée : iatrogénie médicamenteuse, non- 
adhésion, sous-traitement (perte de chances). Ces problèmes sont souvent associés (...) [Elle] met en exergue la nécessité d'une analyse systématique et complète de la prise en charge en interrogeant(i) la qualité de prescription, (ii) les savoir-faire du patient et (iii) la nécessité d'une meilleure coordination pluridisciplinaire et pluriprofessionnelle, les problèmes d'iatrogénie, non-adhésion, sous-traitement/perte de chances n'étant pas exclusifs l'un de l'autre ». Face aux complexités de prise en charge, la coordination pluriprofessionnelle et pluridisciplinaire semble la clé d'une meilleure sécurité et d'un gage de qualité [27]. Concernant les prescriptions, la HAS [28] publie et incite à une meilleure conciliation médicamenteuse afin de sécuriser la prise en charge médicamenteuse du patient lors de son parcours de soins. "La conciliation des traitements médicamenteux est une démarche de prévention et d'interception des erreurs médicamenteuses qui repose sur la transmission et le partage des informations complètes et exactes des traitements du patient entre les professionnels de santé à tous les points de transition. »

\section{Conclusion}

Si la prescription conditionnelle est un espace de liberté pour les infirmiers, elle doit répondre à des règles et des protocoles précis et surtout être évaluée en équipe. Il est essentiel de rappeler l'importance du travail en équipe et l'importance de l'harmonisation des pratiques. La difficulté à cerner la prescription conditionnelle, mais également l'étendue des recommandations sur cette thématique sont intéressantes en termes de questionnement pour les équipes médicales et paramédicales. A l'heure de la coopération médecin/infirmier, il est primordial de connaître les différents apports qui nous informent sur ce que doit être notre travail au quotidien et comment le faire au mieux afin d'assurer une qualité et une sécurité des soins. La personne âgée, de par sa fragilité, incite à prendre en compte certaines précautions, et, il est toujours nécessaire de réévaluer la balance bénéfice/risque en lien avec sa prise en charge.

Conflits d'intérêts : aucun. 


\section{Références}

1-Arrêté du 6 avril 2011 relatif au management de la qualité de la prise en charge médicamenteuse et aux médicaments dans les établissements de santé. https://www.legifrance.gouv.fr/eli/arrete/2011/4/6/ETSH1109848A/jo Consulté le 19/12/2018.

2-Meyer D, Letalon E, Mechin C, et al. Circuit du médicament en établissement de santé : état d'urgence ? Risques \& Qualité 2017; 1: 16-22.

3-DHOS. Prise en charge thérapeutique du patient hospitalisé, 2004. http://www.optimiz-sihcirc-med.fr/Documents/DHOS_circuit_medic_version_publiee.pdf Consulté le 19/12/2018.

4-Chator PE. Evaluation de la prescription conditionnelle en Lorraine : de la réalité hospitalière à la pratique officinale. Sciences pharmaceutiques. 2016. <hal-01731986> https://hal.univ-lorraine.fr/hal-01731986 Consulté le 19/12/2018.

5-DGOS. Qualité de la prise en charge médicamenteuse. Outils pour les établissements de santé, février 2012. http://solidaritessante.gouv.fr/IMG/pdf/Guide_qualite_de_la_prise_en_charge_medicamenteuse.pdf Consulté le $19 / 12 / 2018$.

6-Code de la santé publique - Article R4127-8. https://www.legifrance.gouv.fr/affichCodeArticle.do?idArticle=LEGIARTI000006912869\&ci dTexte=LEGITEXT000006072665\&dateTexte=20100625 Consulté le 19/12/2018.

7-Code de la santé publique. Participation à la protection de la santé. https://www.legifrance.gouv.fr/affichCode.do;jsessionid=35D99C2444E2059A5D9AB14013 18168B.tplgfr27s_2?idSectionTA=LEGISCTA000006198786\&cidTexte=LEGITEXT000006 $072665 \&$ dateTexte $=20180530$ Consulté le 19/12/2018.

8-Code de la santé publique. Actes professionnels. https://www.legifrance.gouv.fr/affichCode.do?idArticle=LEGIARTI000006913888\&idSectio nTA $=$ LEGISCTA000006190610\&cidTexte $=$ LEGITEXT000006072665\&dateTexte $=2018053$ 0 Consulté le 19/12/2018.

9-DGOS. Circulaire $\mathrm{N}^{\circ}$ DGOS/PF2/2012/72 du 14 février 2012 relative au management de la qualité de la prise en charge médicamenteuse dans les établissements de santé. http://circulaire.legifrance.gouv.fr/pdf/2012/02/cir_34700.pdf Consulté le 19/12/2018.

10-Diplôme d'Etat d'Infirmier. Référentiel d'activités. BO Santé - Protection sociale Solidarités no 2009/7 du 15 août 2009. http://solidaritessante.gouv.fr/IMG/pdf/arrete_du_31_juillet_2009_annexe_1.pdf Consulté le 19/12/2018.

11-de Benalcazar S. A la recherche de la nature juridique de la prescription conditionnelle. RGDM $2018 ; 67$ : 149-56.

12-Zarifian P. Le modèle de la compétence. Paris: Editions Liaisons, 2001.

13-Piguet C. Autonomie dans les pratiques infirmières hospitalières, contribution à une théorie agentique du développement professionnel. Education. Université de Nanterre - Paris X, 2008. <tel-00331476> https://tel.archives-ouvertes.fr/tel-00331476/document Consulté le $19 / 12 / 2018$ 
14-Smeulers M, Lucas C, Vermeulen H. Effectiveness of different nursing handover styles for ensuring continuity of information in hospitalised patients. Cochrane Database Syst Rev 2014 Jun 24;(6):CD009979.

15-AFSSAPS. Prévenir la iatrogénèse médicamenteuse chez le sujet âgé, juin 2005. https://ansm.sante.fr/var/ansm_site/storage/original/application/9641eb3f4a1e67ba18a6b8aec d3f1985.pdf Consulté le 19/12/2018.

16-Centres de collaboration nationale en santé publique «Tout éclaircir ». "Qu'est-ce qui constitue une donnée probante ? » Une perspective philosophique, janvier 2010. https://www.ccnpps.ca/docs/Weinstock_DonnéeProbante_Fr.pdf Consulté le 19/12/2018.

17-Kahl C, Meirelles BHS, Lanzoni GMM, et al. Actions and interactions in clinical nursing practice in Primary Health Care. Rev Esc Enferm USP. 2018;52:e03327.

18-Aubin M, Giguère A, Martin M, et al. Interventions to improve the continuity of care in the follow-up of patients with cancer. Cochrane Database Syst Rev. 2012 Jul 11;(7):CD007672.

19-Dancausse F, Chaumat E. Les transmissions ciblées au service de la qualité des soins. Issyles-Moulineaux, Elsevier-Masson (3e édition), 2008 : 161-76.

20-Laurens C. Les interactions soignantes à l'hôpital. La relève interéquipe moment clé de la communication. Paris, Éditions Seli Arslan, 2016 : 82.

21-Grosjean M, Lacoste M. Communication et intelligence collective. Le travail à l'hôpital. Paris, PUF, 1999 : 77.

22-Laurent A, Capellier G. Accompagner les professionnels de santé dans la révélation de l'erreur : de la révélation à soi à la révélation à l'autre. Ann Med Psychol 2018; 176 : 145-9.

23-Kramer M, Schmalenberg CE. Magnet hospital staff nurses describe clinical autonomy. Nurs Outlook 2003; 51: 13-9.

24-Vera M, Martınez IM, Lorente L, et al. The role of co-worker and supervisor support in the relationship between job autonomy and work engagement among portuguese nurses: A multilevel study. Soc Indic Res 2016; 126: 1143-56.

25-Wade GH. Professional nurse autonomy: Concept analysis and application to nursing education. J Adv Nurs 1999; 30(2): 310-8.

26-Rao AD, Kumar A, McHugh M. Better nurse autonomy decreases the odds of 30-day mortality and failure to rescue. J Nurs Scholarsh 2017;49(1):73-9.

27-HAS. Comment améliorer la qualité et la sécurité des prescriptions de médicaments chez la personne âgée ?, septembre 2014. https://www.hassante.fr/portail/jcms/c_1771494/fr/ameliorer-la-qualite-et-la-securite-des-prescriptions-demedicaments-chez-la-personne-agee-note-methodologique Consulté le 19/12/2018.

28-HAS. Mettre en œuvre la conciliation des traitements médicamenteux en établissement de santé. Sécuriser la prise en charge médicamenteuse du patient lors de son parcours de soins, février 2018. https://www.has-sante.fr/portail/jcms/c_2736442/fr/mettre-en-oeuvre-laconciliation-des-traitements-medicamenteux-en-etablissement-de-sante Consulté le 19/12/2018. 
Tableau 1: Proposition de bonnes pratiques et recommandations professionnelles (adaptées de Chator [4]).

\section{Pour la prescription (médecin)}

- Une voie d'administration, une posologie ainsi qu'une durée de validité.

- Un motif de mise en place justifié.

- Une ou des conditions d'administration clairement établies voire protocolisées.

- Un nombre maximal d'administrations par période de 24 heures accompagné d'un intervalle temporel entre deux prises consécutives.

- Un traitement de fond, adapté à la symptomatologie ciblée par les administrations conditionnelles.

- Un planning de réévaluation de la pertinence de cette prescription sera fixé au moment même de la dite prescription.

\section{Pour la dispensation (pharmacien)}

Concernant la dispensation de ce type de prescription :

- $\quad$ En l'absence de toute notion de durée, le pharmacien doit dispenser le conditionnement représentant la plus petite quantité.

- $\quad$ Si à l'inverse, un cadrage temporel est fixé, le pharmacien est en droit de dispenser la quantité suffisante pour couvrir l'ensemble des administrations éventuelles.

Néanmoins le dispensateur devra s'efforcer de déterminer par le biais d'un dialogue avec le patient la quantité optimale nécessaire pour subvenir aux besoins réels de celui-ci. Tout en accompagnant la dispensation de la spécialité de conseils attenant aux conditions d'administration et à l'auto-évaluation de l'efficacité du traitement proposé.

\section{Pour l'administration et le suivi (infirmier)}

- La traçabilité de l'administration (ou non administration) ainsi que l'efficacité du traitement devront être retrouvées dans le dossier du patient (qu'il soit informatique ou non).

- $\quad$ En complément de ces mesures, l'initiation d'une prescription conditionnelle d'antalgique devra, dans la mesure du possible, s'inscrire dans un protocole de soins en collaboration étroite avec le personnel infirmier. 